\title{
Factorization of the transfer matrices for the quantum $s \ell(2)$ spin chains and Baxter equation
}

\author{
S É Derkachov ${ }^{1}, \quad$ A N Manashov ${ }^{2} \ddagger$ \\ ${ }^{1}$ St.Petersburg Department of Steklov Mathematical Institute of Russian Academy of Sciences, \\ Fontanka 27, 191023 St.Petersburg, Russia. \\ ${ }^{2}$ Institute for Theoretical Physics, University of Regensburg, \\ D-93040 Regensburg, Germany
}

\begin{abstract}
It is shown that the transfer matrices of homogeneous $s \ell(2)$ invariant spin chains with generic spin, both closed and open, are factorized into the product of two operators. The latter satisfy the Baxter equation that follows from the structure of the reducible representations of the $s \ell(2)$ algebra.
\end{abstract}




\section{Introduction}

The recent interest to the analysis of noncompact spin magnets (spin chains with the infinite dimensional Hilbert space at each site) is motivated by the advances in gauge field theories (see for a review [1, 2]). These models (spin magnets) can be solved with the help of the Algebraic Bethe Ansatz (ABA) method [3, 4]. Alternatively, the solution is provided by the method of the Baxter $Q$-operators [5].

The Baxter $Q$-operator is known for a large number of integrable models [6, 7, 8, 9, 10, 11, 12, 13, 14, 15. Nevertheless, a universal method for obtaining of the Baxter operator is absent so far and each model (or class of models) needs a special consideration. The derivation of the Baxter $Q$-operator for the $s \ell(2)$ spin chain models is based on the Pasquier-Gaudin trick, see Refs. 6, 9, 16. The generalization of the latter to the spin chains with the higher rank symmetry groups is not quite obvious.

In the present paper we give the alternative derivation of the Baxter equation for the noncompact $X X X$ spin chain models. We shall show that the transfer matrices for the homogeneous spin chain models factorize into a product of two operators. The factorization holds for all closed $s \ell(2)$ spin chains studied so far [16, 14, 17] and can be traced to the factorization of the $\mathcal{R}$-operator obtained in Ref. [18. We prove that this property is true for the open spin chain models as well. As the factorization property is established, the Baxter equation for these operators can be deduced in a simple way from the structure of the reducible representations of the $s \ell(2)$ algebra. (See Ref. 19. where similar arguments were applied to the analysis of $q$-deformed spin chain models.) We shall consider the spin chains with the quantum space being the generic lowest weight representation of the $s \ell(2)$ algebra, but the method works for the principal series representations of the $S L(2, \mathbb{R})$ $(S L(2, \mathbb{C}))$ group as well. Taking into account that, as it was shown in Ref. 18, factorization holds for the $s \ell(3)$ and $s \ell(2 \mid 1)$ invariant $\mathcal{R}$-operators one can hope that the approach presented here admits a generalization for the spin chains with the symmetry group of higher rank.

The paper is organized as follows: In Section 2 we introduce notations and describe the model. In Section 3 we prove the factorization property for the transfer matrices for both the closed and open $s \ell(2)$ noncompact spin chain models. In Section 4 the derivation of the Baxter equation based on the structure of the reducible $s \ell(2)$ representations is given. Section 5 contains concluding remarks.

\section{Preliminaries}

The basic object in the theory of the lattice integrable systems is a $\mathcal{R}$-operator. The $\mathcal{R}$ - operator is a linear operator which depends on a spectral parameter $u$ and acts on the tensor product of two $s \ell(2)$ modules (representations of the $s \ell(2)$ algebra). It satisfies the Yang-Baxter relation (YBR)

$$
\mathcal{R}_{12}(u) \mathcal{R}_{13}(u+v) \mathcal{R}_{23}(v)=\mathcal{R}_{23}(v) \mathcal{R}_{13}(u+v) \mathcal{R}_{12}(u) .
$$

The operators acts on the tensor product $\mathbf{V}_{1} \otimes \mathbf{V}_{2} \otimes \mathbf{V}_{3}$, and, as usual, indices $i k$ indicate that the operator $\mathcal{R}_{i k}$ acts nontrivially on the tensor product $\mathbf{V}_{i} \otimes \mathbf{V}_{k}$. We shall consider the $s \ell(2)$ invariant solutions of the YBR.

The $s \ell(2)$ algebra has three generators $S_{+}, S_{-}$and $S_{0}$ which satisfy the well known commutation relations

$$
\left[S_{0}, S_{ \pm}\right]= \pm S_{ \pm}, \quad\left[S_{+}, S_{-}\right]=2 S_{0} .
$$

The lowest weight representation of $s \ell(2)$ algebra, $D_{s}$, is uniquely determined by the complex number ( $\operatorname{spin}$ ) $s$. The generators can be realized as the differential operators

$$
S_{-}=-\partial_{z}, \quad S_{+}=z^{2} \partial_{z}+2 s z, \quad S_{0}=s \partial_{z}+s
$$


acting on the linear space $\mathbf{V}_{s}=\mathbb{C}[z]$ (the space of polynomials of arbitrary degree of a complex variable $z$ ). For a given $s$ the representation (2.3) is irreducible unless $s$ is a negative (half)integer. If $s=-n, n=0,1 / 2,1, \ldots$ the space $\mathbf{V}_{s}$ contains a finite dimensional invariant subspace, $V_{n}$, the space of polynomials of degree less or equal to $2 n,\left(\operatorname{dim} V_{n}=2 n+1\right)$. The representation induced on the factor space $\mathbf{V}_{-n} / V_{n}$ is equivalent to the representation $D_{s^{\prime}}$ with spin $s^{\prime}=1+n$. The operator A which intertwines the representations $D_{-n}$ and $D_{n+1},\left(\mathrm{~A} D_{-n}=D_{1+n} \mathrm{~A}\right)$, is defined by the commutation relations

and has the form $\mathrm{A}=\partial_{z}^{2 n+1}$.

$$
\mathrm{A} S_{\alpha}^{(s=-n)}=S_{\alpha}^{(s=n+1)} \mathrm{A}
$$

For the real $s>1 / 2$ there exists the invariant scalar product $(\cdot, \cdot)_{s}$ on the space $V_{s}$,

$$
\left(\psi_{1}, \psi_{2}\right)_{s}=\int \mathcal{D}_{s} z \overline{\psi_{1}(z)} \psi_{2}(z)
$$

where

$$
\int \mathcal{D}_{s} z \varphi(z, \bar{z}) \equiv \frac{2 s-1}{\pi} \int_{|z|<1} d^{2} z\left(1-|z|^{2}\right)^{2 s-2} \varphi(z, \bar{z}) .
$$

The operator $S_{0}$ is hermitian with respect to the scalar product (2.4), while $S_{-}^{\dagger}=-S_{+}$. For complex $s$ the integral (2.4) defines the invariant bilinear form on the tensor product $\mathbf{V}_{s^{*}} \otimes \mathbf{V}_{s}$. The unit operator (reproducing kernel) has the form

$$
\mathbb{K}_{s}(z, w)=(1-z \bar{w})^{-2 s} .
$$

The identity

$$
\psi(z)=\int \mathcal{D}_{s} w \mathbb{K}_{s}(z, w) \psi(w),
$$

where $\psi(w)$ is the function analytic in the unit circle holds for complex $s$ such that $\operatorname{Re} s>1 / 2$; for all other spins it should be understood as an analytic continuation in $s$.

The $s \ell(2)$ invariant $\mathcal{R}$-operator acting on the tensor product of two spaces $\mathbf{V}_{s_{1}} \otimes \mathbf{V}_{s_{2}}$ has the form 20, 4, 21]

$$
\mathcal{R}_{12}(u)=(-1)^{\mathbb{J}-s_{1}-s_{2}} \frac{\Gamma\left(s_{1}+s_{2}+i u\right)}{\Gamma\left(s_{1}+s_{2}-i u\right)} \frac{\Gamma(\mathbb{J}-i u)}{\Gamma(\mathbb{J}+i u)},
$$

where the operator of the conformal spin $\mathbb{J}$ is related to the two-particle Casimir operator in the standard manner

$$
\mathbb{J}(\mathbb{J}-1)=\left(\vec{S}_{1}+\vec{S}_{2}\right)^{2} .
$$

It was shown in the Ref. [18] that the $\mathcal{R}$-operator (2.8) can be represented in the factorized form

$$
\mathcal{R}_{12}(u)=P_{12} \mathcal{R}_{12}^{+}(\alpha) \mathcal{R}_{12}^{-}(\beta)=P_{12} \mathcal{R}_{12}^{-}(\beta) \mathcal{R}_{12}^{+}(\alpha) .
$$

Here $P_{12}$ is the permutation operator $P_{12} \psi\left(z_{1}, z_{2}\right)=\psi\left(z_{2}, z_{1}\right)$, and

$$
\alpha=\frac{s_{2}-s_{1}+i u}{2}, \quad \beta=\frac{s_{1}-s_{2}+i u}{2} .
$$

The operator $\mathcal{R}_{12}^{-}(\alpha)$ is a $s \ell(2)$ covariant operator, i.e. it maps

$$
\mathbf{V}_{s_{1}} \otimes \mathbf{V}_{s_{2}} \rightarrow \mathbf{V}_{s_{1}-\alpha} \otimes \mathbf{V}_{s_{2}+\alpha}
$$

and has the following form

$$
\mathcal{R}_{12}^{-}(\alpha)=\frac{\Gamma\left(2 s_{1}\right)}{\Gamma\left(2 s_{1}-2 \alpha\right)} \frac{\Gamma\left(z_{12} \partial_{1}+2 s_{1}-2 \alpha\right)}{\Gamma\left(z_{12} \partial_{1}+2 s_{1}\right)},
$$




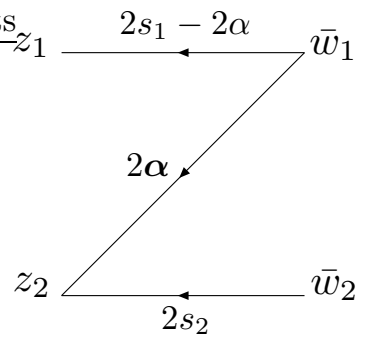

Figure 1. Graphical representation of the $\mathcal{R}_{12}^{-}(\alpha)$-operator. The arrow with the index $\alpha$ directed from $\bar{w}$ to $z$ denotes the factor $(1-z \bar{w})^{-\alpha}$.

where $z_{12}=z_{1}-z_{2}$. Such normalization implies that $R_{12}^{-}(0)=\mathbb{I}$ and $R_{12}^{-}(\alpha) \cdot 1=1$. The second operator, $\mathcal{R}_{12}^{+}(\alpha),\left(\mathcal{R}_{12}^{+}(\alpha): \mathbf{V}_{s_{1}} \otimes \mathbf{V}_{s_{2}} \rightarrow \mathbf{V}_{s_{1}+\alpha} \otimes \mathbf{V}_{s_{2}-\alpha}\right)$ is

$$
\mathcal{R}_{12}^{+}(\alpha)=\mathcal{R}_{21}^{-}(\alpha)=\frac{\Gamma\left(2 s_{2}\right)}{\Gamma\left(2 s_{2}-2 \alpha\right)} \frac{\Gamma\left(z_{21} \partial_{2}+2 s_{2}-2 \alpha\right)}{\Gamma\left(z_{21} \partial_{2}+2 s_{2}\right)} .
$$

The operators $\mathcal{R}_{12}^{ \pm}(\alpha)$ depend on three parameters - the spins $s_{1}, s_{2}$ and the spectral parameter $\alpha$. The spins are always fixed by the tensor properties of the space $\mathbf{V}_{s_{1}} \otimes \mathbf{V}_{s_{2}}$ the operators act on, therefore we shall display the dependence of the operators on the spectral parameter only. The action of the $\mathcal{R}$-operator (2.10) on the space $\mathbf{V}_{s_{1}} \otimes \mathbf{V}_{s_{2}}$ results in the following chain of transformations

$$
\mathbf{V}_{s_{1}} \otimes \mathbf{V}_{s_{2}} \stackrel{\mathcal{R}_{12}^{-}(\beta)}{\longrightarrow} \mathbf{V}_{\left(s_{1}+s_{2}-i u\right) / 2} \otimes \mathbf{V}_{\left(s_{1}+s_{2}+i u\right) / 2} \stackrel{\mathcal{R}_{12}^{+}(\alpha)}{\longrightarrow} \mathbf{V}_{s_{2}} \otimes \mathbf{V}_{s_{1}} \stackrel{P_{12}}{\longrightarrow} \mathbf{V}_{s_{1}} \otimes \mathbf{V}_{s_{2}}
$$

In the next section we shall represent the operators $\mathcal{R}_{12}^{ \pm}(\alpha)$ as integral operators and prove the factorization of the transfer matrices 3,22

$$
\begin{aligned}
& \mathbf{T}_{s_{0}}^{\mathrm{cl}}=\operatorname{tr}_{s_{0}} \mathcal{R}_{10}(u) \ldots \mathcal{R}_{N 0}(u), \\
& \mathbf{T}_{s_{0}}^{\mathrm{op}}=\operatorname{tr}_{s_{0}} \mathcal{R}_{10}(u) \ldots \mathcal{R}_{N 0}(u) \mathcal{R}_{N 0}^{-1}(-u) \ldots \mathcal{R}_{10}^{-1}(-u)
\end{aligned}
$$

for the homogeneous closed and open $s \ell(2)$ invariant spin chains. The $\mathcal{R}$-operator obeys the relation $\mathcal{R}_{12}^{-1}(u)=\mathcal{R}_{12}(-u)$ so that we shall use the following expression for the $\mathbf{T}_{s_{0}}^{\mathrm{op}}$

$$
\mathbf{T}_{s_{0}}^{\mathrm{op}}=\operatorname{tr}_{s_{0}} \mathcal{R}_{10}(u) \ldots \mathcal{R}_{N 0}(u) \mathcal{R}_{N 0}(u) \ldots \mathcal{R}_{10}(u) .
$$

\section{Factorization}

We find convenient to represent all operators in question as integral operators. Let us write the action of the operator $\mathcal{A}$ on the function $\psi \in \prod_{k=1}^{N} \otimes \mathbf{V}_{s_{k}}$ in the following form

$$
[\mathcal{A} \psi](\boldsymbol{z})=\int \prod_{k=1}^{N} \mathcal{D}_{s_{k}} w_{k} A(\boldsymbol{z} \mid \overline{\boldsymbol{w}}) \psi(\boldsymbol{w}),
$$

where $\boldsymbol{z}=\left(z_{1}, \ldots, z_{N}\right)$. It follows from the definition (3.1) and Eq. (2.7) that the kernel of the operator $\mathcal{A}$ can be obtained as follows

$$
A(\boldsymbol{z} \mid \overline{\boldsymbol{w}})=\mathcal{A} \cdot \prod_{k=1}^{N}\left(1-z_{k} \bar{w}_{k}\right)^{-2 s_{k}} .
$$

Here the operator $\mathcal{A}$ on the r.h.s of Eq. (3.2) acts on $\boldsymbol{z}$-variables.

It is easy to show that the kernel of the operator $\mathcal{R}_{12}^{-}(\alpha)$ takes the following form

$R_{12}^{-}(\alpha)\left(z_{1}, z_{2} \mid \bar{w}_{1}, \bar{w}_{2}\right)=\left(1-z_{1} \bar{w}_{1}\right)^{-2 s_{1}+2 \alpha}\left(1-z_{2} \bar{w}_{1}\right)^{-2 \alpha}\left(1-z_{2} \bar{w}_{2}\right)^{-2 s_{2}}$. 

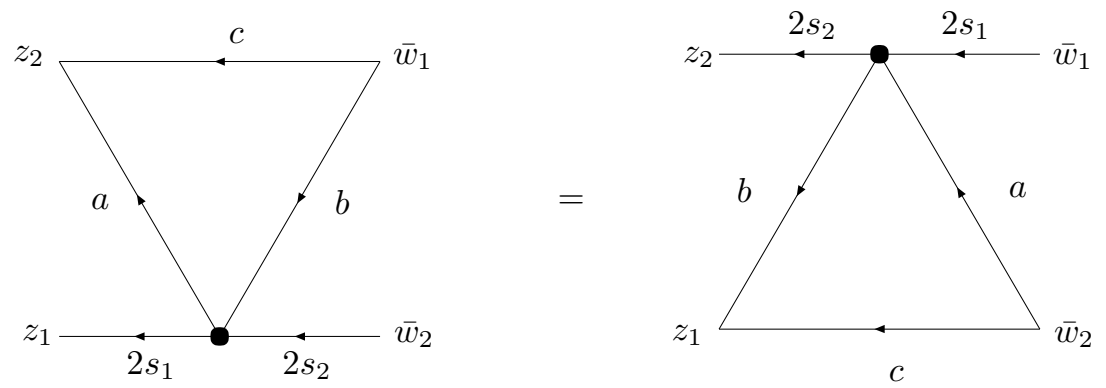

Figure 2. The two equivalent graphical representation of the kernel of the $\mathcal{R}$ - operator. The black dot denotes the integration vertex with the measure corresponding to the spin $\left(s_{1}+s_{2}+i u\right) / 2$ and the indicies $a=2 \alpha=s_{2}-s_{1}+i u, b=2 \beta=s_{1}-s_{2}+i u, c=2 \gamma=s_{1}+s_{2}-i u$.

It is convenient to represent the kernel $R_{12}^{-}(\alpha)(\boldsymbol{z} \mid \overline{\boldsymbol{w}})$ in the graphical form. Namely, let us denote the reproducing kernel $\mathbb{K}_{\alpha}(z, w)=(1-z \bar{w})^{-2 \alpha}$ by the arrow with the index $2 \alpha$ directed from $w$ to $z$. Then the kernel $R_{12}^{-}(\alpha)(\boldsymbol{z} \mid \overline{\boldsymbol{w}})$ is given by the diagram shown in the Figure 1 Similarly, as follows from Eq. (2.10), the kernel of the $\mathcal{R}_{12}$-operator has the form

$$
\begin{aligned}
R_{u}\left(z_{1}, z_{2} \mid \bar{w}_{1}, \bar{w}_{2}\right)= & \left(1-z_{2} \bar{w}_{1}\right)^{-2 \gamma} \int D_{\left(s_{1}+s_{2}+i u\right) / 2} \zeta \\
& \left(1-z_{1} \bar{\zeta}\right)^{-2 s_{1}}\left(1-z_{2} \bar{\zeta}\right)^{-2 \alpha}\left(1-\zeta \bar{w}_{1}\right)^{-2 \beta}\left(1-\zeta \bar{w}_{2}\right)^{-2 s_{2}}
\end{aligned}
$$

where $\alpha$ and $\beta$ are defined in Eq. (2.11) and $\gamma=\left(s_{1}+s_{2}-i u\right) / 2$.

There exists another equivalent representation for the $\mathcal{R}$-operator which follows from the second equality in Eq. (2.10). Again, it is useful to represent both of them in the graphical form, see Figure 2 The identity depicted in Figure 2 (permutation relation) can be considered as an integral identity between the reproducing kernels. It will be quite useful in the subsequent analysis.

Let us summarize the properties of the $\mathcal{R}^{-}$-operators. One easily checks that

$$
\begin{aligned}
& \mathcal{R}_{12}^{ \pm}(\alpha) \mathcal{R}_{12}^{ \pm}(\beta)=\mathcal{R}_{12}^{ \pm}(\alpha+\beta), \\
& \mathcal{R}_{12}^{+}\left(s_{2}-s_{1}+\alpha\right) \mathcal{R}_{12}^{-}(\alpha)=\mathcal{R}_{12}^{-}(\alpha) \mathcal{R}_{12}^{+}\left(s_{2}-s_{1}+\alpha\right), \\
& \mathcal{R}_{12}^{ \pm}(\alpha) \mathcal{R}_{23}^{ \pm}(\alpha+\beta) \mathcal{R}_{12}^{ \pm}(\beta)=\mathcal{R}_{23}^{ \pm}(\beta) \mathcal{R}_{12}^{ \pm}(\alpha+\beta) \mathcal{R}_{23}^{ \pm}(\alpha) .
\end{aligned}
$$

The first equality follows from Eq. (3.3) and from the property of the reproducing kernel. The second one is the consequence of Eq. (2.10). The last one arises as the selfconsistency relation of the defining equations for the $\mathcal{R}^{ \pm}$operators [17] and can be checked directly by making use of the permutation relation.

Let us introduce operators $\mathcal{L}_{12}^{ \pm}(\alpha)=P_{12} \mathcal{R}_{12}^{ \pm}(\alpha)$. It is straightforward to check that these operators satisfy the relation

$$
\mathcal{L}_{12}^{ \pm}(\alpha) \mathcal{L}_{13}^{ \pm}(\alpha+\beta) \mathcal{L}_{23}^{ \pm}(\beta)=\mathcal{L}_{23}^{ \pm}(\beta) \mathcal{L}_{13}^{ \pm}(\alpha+\beta) \mathcal{L}_{12}^{ \pm}(\alpha) .
$$

Equation (3.8) has the form of the Yang-Baxter relation, but in difference to the $\mathcal{R}$-operator, the operators $\mathcal{L}_{12}^{ \pm}(\alpha)$ map the space $\mathbf{V}_{s_{1}} \otimes \mathbf{V}_{s_{2}} \mapsto \mathbf{V}_{s_{2} \pm \alpha} \otimes \mathbf{V}_{s_{1} \mp \alpha}$. However, for the special values of the spectral parameter, $\alpha_{ \pm}= \pm\left(s_{2}-s_{1}\right)$, the operators $\mathcal{L}_{12}^{ \pm}\left(\alpha_{ \pm}\right)$coincide with the $\mathcal{R}$-operator for the special values of the spectral parameter,

$$
\mathcal{L}_{12}^{ \pm}\left( \pm\left(s_{2}-s_{1}\right)\right)=\mathcal{R}_{12}\left(\mp i\left(s_{2}-s_{1}\right)\right),
$$

and play an important role in the subsequent construction. 
Factorization of the transfer matrices

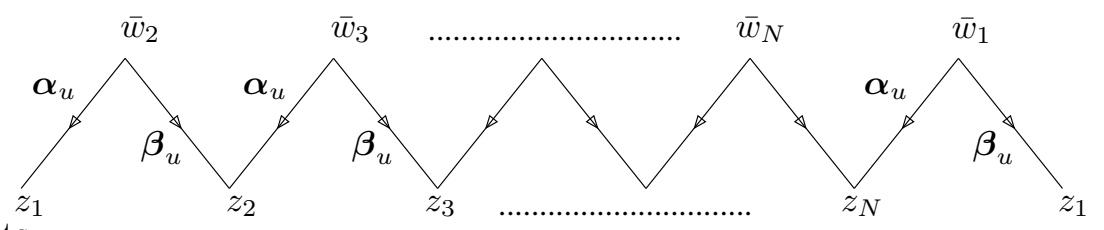

Figure 3. The graphical representation of the kernel of $Q(u)$ operator for the closed spin chain; $\alpha_{u}=s-i u$ and $\beta_{u}=s+i u$.

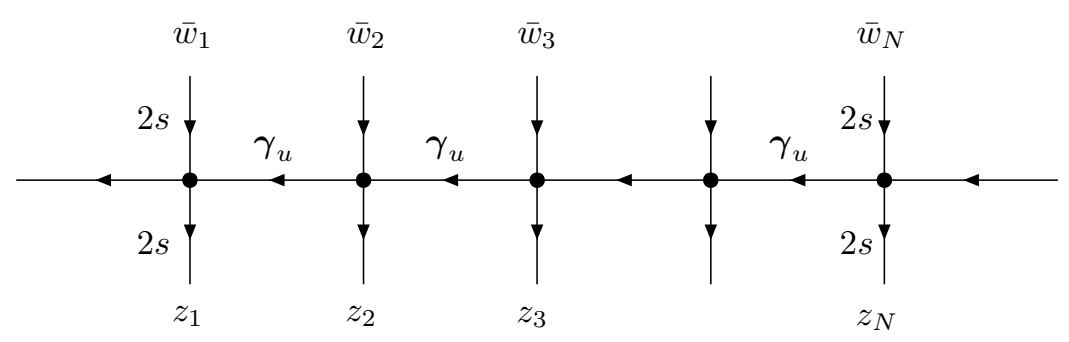

Figure 4. The kernel of the $\widetilde{Q}(u)$ operator for the closed spin chain. All horizontal lines carry the index $\gamma_{u}=i u-s$, while the vertical ones have the index $2 s$. The black dots denote the integration with the measure corresponding to the spin $s^{\prime}=(s+i u) / 2$.

In what follows we show that the transfer matrix for the closed homogeneous spin chain (2.16) can be represented in the factorized form

$\mathbf{T}_{s_{0}}^{\mathrm{cl}}(u)=Q\left(u+i s_{0}\right) \widetilde{Q}\left(u-i s_{0}\right)=\widetilde{Q}\left(u-i s_{0}\right) Q\left(u+i s_{0}\right)$,

where $s \ell(2)$ invariant $Q$-operators are given by the traces of $\mathcal{L}_{12}^{ \pm}$operators. Namely, we get

$$
\begin{aligned}
& Q(u)=\left.\operatorname{tr}_{s_{0}} \mathcal{L}_{10}^{-}\left(s-s_{0}\right) \ldots \mathcal{L}_{N 0}^{-}\left(s-s_{0}\right)\right|_{s_{0}=(s-i u) / 2}, \\
& \widetilde{Q}(u)=\left.\mathcal{P}_{\operatorname{tr}_{s_{0}}} \mathcal{L}_{10}^{+}\left(s_{0}-s\right) \ldots \mathcal{L}_{N 0}^{+}\left(s_{0}-s\right)\right|_{s_{0}=(s+i u) / 2},
\end{aligned}
$$

where $\mathcal{P}$ is the cyclic permutation operator, $\mathcal{P} \psi\left(z_{1}, z_{2}, \ldots, z_{N}\right)=\psi\left(z_{2}, z_{3}, \ldots, z_{1}\right)$. Taking into account (3.9) we conclude that the operators $Q(u)$ and $\widetilde{Q}(u)$ coincide with the transfer matrices

$$
\begin{aligned}
& Q(u)=\mathbf{T}_{(s-i u) / 2}\left(\frac{u-i s}{2}\right), \\
& \widetilde{Q}(u)=\mathcal{P} \mathbf{T}_{(s+i u) / 2}\left(\frac{u+i s}{2}\right) .
\end{aligned}
$$

The commutativity of the $Q, \widetilde{Q}$ operators, $[Q(u), Q(v)]=[\widetilde{Q}(u), \widetilde{Q}(v)]=[Q(u), \widetilde{Q}(v)]=0$, follows immediately from the commutativity of the transfer matrices, (we remind that $\mathcal{P}^{-1}=\mathbf{T}_{s}^{s}(0)$.)

Making use of Eqs. (3.13), (3.14) one can represent Eq. (3.10) in the following form

$\mathbf{T}_{s_{0}}(u)=\mathbf{T}_{\left(s+s_{0}-i u\right) / 2}\left(\frac{u-i\left(s-s_{0}\right)}{2}\right) \mathcal{P} \mathbf{T}_{\left(s+s_{0}+i u\right) / 2}\left(\frac{u+i\left(s-s_{0}\right)}{2}\right)$.

To prove the factorization property we shall show that the integral kernels of the operators on the l.h.s and r.h.s of Eq. (3.10) coincide. To this end let us represent the kernel of the operators under consideration in the graphical form. The diagrammatical representation of the kernels for the operators $Q(u)$ and $\widetilde{Q}(u)$ are shown in Figures 3 and 4 respectively. In its turn, the integral kernel for the transfer matrix is shown, in two equivalent forms, in Figure 5 Drawing the diagram for the product $Q\left(u+i s_{0}\right) \widetilde{Q}\left(u-i s_{0}\right)$ (or $\left.\widetilde{Q}\left(u-i s_{0}\right) Q\left(u+i s_{0}\right)\right)$ one notices that the measure of integration 


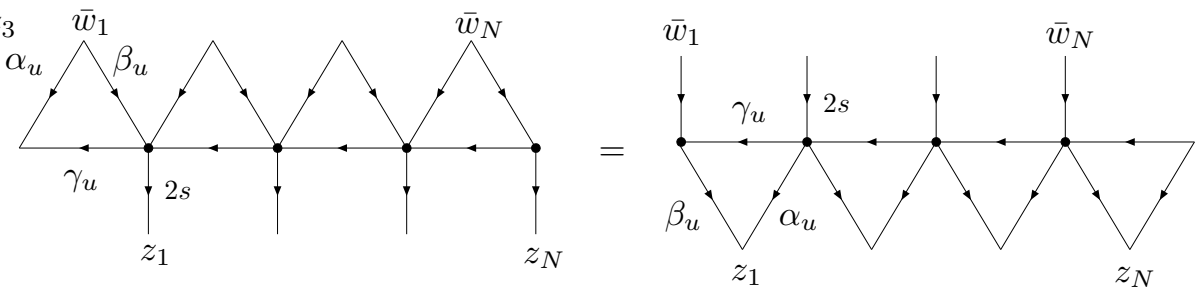

Figure 5. The graphical representation of the transfer matrix for the closed spin chain. The indices $\alpha_{u}=s-i\left(u+i s_{0}\right), \beta_{u}=s+i\left(u+i s_{0}\right)$. The indices of the vertical lines are equal to $2 s$, and those of the horizontal are equal to $\gamma_{u}=i\left(u-i s_{0}\right)-s$. The black dots denotes the integration vertices corresponding to the spin $s^{\prime}=\left(s+i\left(u-i s_{0}\right)\right) / 2$.

in the intermediate triple vertices corresponds to the spin $s$. Since the vertical lines attached to this vertex correspond to the reproducing kernel with the spin $s$, one can carry out the integration using the property (2.7) and find that the resulting diagram coincides with the diagram for the kernel of the transfer matrix. Thus the property of the factorization for the homogenous spin chain is established.

For completeness, we write down the analytic expressions for the kernels of the $Q$-operators,

$Q(u)(\boldsymbol{z} \mid \boldsymbol{w})=\prod_{k=1}^{N}\left(1-z_{k} \bar{w}_{k}\right)^{-s-i u}\left(1-z_{k} \bar{w}_{k+1}\right)^{-s+i u}$,

$\widetilde{Q}(u)(\boldsymbol{z} \mid \boldsymbol{w})=\prod_{k=1}^{N} \int \mathcal{D}_{s^{\prime}} \zeta_{k}\left(1-\zeta_{k} \bar{\zeta}_{k+1}\right)^{s-i u}\left(1-z_{k} \bar{\zeta}_{k}\right)^{-2 s}\left(1-\zeta_{k} \bar{w}_{k}\right)^{-2 s}$,

where $s^{\prime}=s+i u / 2$, and $w_{N+1} \equiv w_{1}$ and so on. The expression (3.16) coincides with the expression for the Baxter operator obtained in Ref. [9].

Let us consider now the homogeneous $s \ell(2)$ invariant open spin chain. The transfer matrix for the open spin chain, (2.16), can also be represented in the factorized form, namely

$\mathbf{T}_{s_{0}}^{\mathrm{op}}(u)=g(u) \mathcal{Q}\left(u+i s_{0}\right) \widetilde{\mathcal{Q}}\left(u-i s_{0}\right)=g(u) \widetilde{\mathcal{Q}}\left(u-i s_{0}\right) \mathcal{Q}\left(u+i s_{0}\right)$,

where

$$
g(u)=\frac{s+s_{0}+i u-1}{2 i u-1} .
$$

The operators $\mathcal{Q}(u)$ and $\widetilde{\mathcal{Q}}(u)$ have the following form

$\mathcal{Q}(u)=\left.\operatorname{tr}_{s_{0}} \mathcal{L}_{10}^{-}\left(s-s_{0}\right) \ldots \mathcal{L}_{N 0}^{-}\left(s-s_{0}\right) \mathcal{L}_{N 0}^{-}\left(s-s_{0}\right) \ldots \mathcal{L}_{10}^{-}\left(s-s_{0}\right)\right|_{s_{0}=\frac{s-i u}{2}}$,

$\widetilde{\mathcal{Q}}(u)=\left.\operatorname{tr}_{s_{0}} \mathcal{L}_{10}^{+}\left(s_{0}-s\right) \ldots \mathcal{L}_{N 0}^{+}\left(s_{0}-s\right) \mathcal{L}_{N 0}^{+}\left(s_{0}-s\right) \ldots \mathcal{L}_{10}^{+}\left(s_{0}-s\right)\right|_{s_{0}=\frac{s+i u}{2}}$.

Again, taking into account Eq. (3.9) one relates $\mathcal{Q}$-operators to the transfer matrices for the open spin chain

$$
\begin{aligned}
& \mathcal{Q}(u)=\mathbf{T}_{(s-i u) / 2}\left(\frac{u-i s}{2}\right), \\
& \widetilde{\mathcal{Q}}(u)=\mathbf{T}_{(s+i u) / 2}\left(\frac{u+i s}{2}\right) .
\end{aligned}
$$

Thus, similarly to the closed spin chain, one concludes that $\mathcal{Q}$-operators commute with each other for arbitrary values of the spectral parameters. 


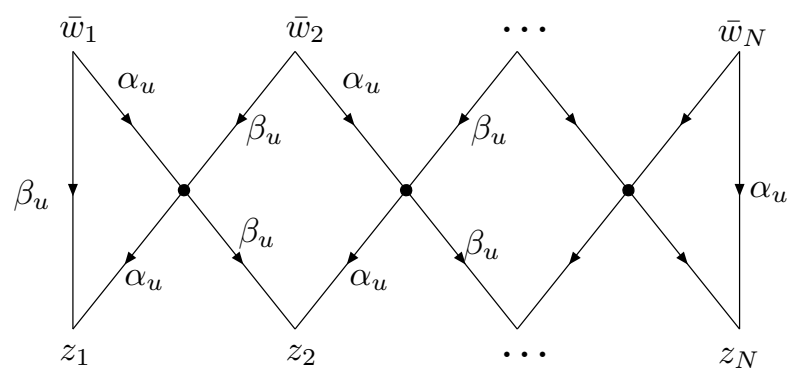

Figure 6. Diagrammatical representation of the $\mathcal{Q}(u)$-operator for the open spin chain; $\alpha_{u}=s-i u$ and $\beta_{u}=s+i u$.



Figure 7. Diagrammatical representation of the $\widetilde{\mathcal{Q}}(u)$-operator for the open spin chain. Here the all horizontal lines carry the index $\gamma_{u}=i u-s$, all vertical lines have index $2 s$. The black dots denote the integration with the measure corresponding to the conformal spin $s^{\prime}=(s+i u) / 2$.

To prove the factorization (3.18) we again use the graphical representation for kernels. For the $\mathcal{Q}(u)$ and $\widetilde{\mathcal{Q}}(u)$ operators they are shown in Figure [6] and 7 respectively. The diagrammatical representation for the kernel of the transfer matrix for the open spin chain is shown in Figure 8 In order to derive this representation one starts with the definition 2.16) and uses the graphical representation for the kernel of $\mathcal{R}$-operator shown on the lhs of Figure 2 Next one should carry out the integration over all intermediate "quantum" vertices $\zeta_{1}, \ldots, \zeta_{N}$. The integration measure in each vertex is given by the expression (2.5) where $s$ is the "spin" of the quantum space. The result of the integration is the disappearance of the lines with index $2 s$

$\int D_{s}^{\prime} \xi \int \mathcal{D} \zeta_{s} \psi(\xi, \bar{\xi}) \mathbb{K}_{s}(\xi, \zeta) \phi(\zeta)=\int D_{s}^{\prime} \xi \psi(\xi, \bar{\xi}) \phi(\xi)$

attached to these vertices. Finally one can carry out the integration over "auxiliary space" vertices. Again, the lines attached to these vertices disappear. The line with the index $2 i u$ (on the right part of the diagram) arises due to merging of two lines with indices $\gamma_{u}$ and $\beta_{u},(1-z \bar{w})^{-\gamma_{u}}(1-z \bar{w})^{-\beta_{u}}=$ $(1-z \bar{w})^{-2 i u}$.

To explain the appearance of the factor $g(u)$ and the integration vertex with spin $s^{\prime \prime}=i u$, we notice that after the integration one line with index $\alpha_{u}$ becomes attached to the vertex with the spin $\left.s^{\prime}=s+i\left(u-i s_{0}\right)\right)$ by both ends. Noticing that

$$
\int D_{s^{\prime}} \xi(1-\xi \bar{\xi})^{-\alpha_{u}} \ldots=g(u) \int D_{i u} \xi \ldots
$$






Figure 8. The graphical representation of the transfer matrix for the open spin chain. Here $\alpha_{u}=s-i\left(u+i s_{0}\right), \beta_{u}=s+i\left(u+i s_{0}\right), \gamma_{u}=i\left(u-i s_{0}\right)-s$, The black dots denotes the integration vertices corresponding to the spin $s^{\prime}=\left(s+i\left(u-i s_{0}\right)\right) / 2$ and white dot denotes the integration vertex corresponding to the spin $s^{\prime \prime}=i u$. The prefactor $g(u)$ is given by Eq. 3.19).

one obtains finally the diagrammatic representation for the kernel of the transfer matrix shown in Figure 8

Now we have to show that the diagram for the transfer matrix can be transformed to the diagram for the product of the operators $\mathcal{Q}\left(u+i s_{0}\right) \widetilde{\mathcal{Q}}\left(u-i s_{0}\right)$. The first transformation is the insertion of the reproducing kernels into the diagram in Fig. 8 as shown in Figure 9 This operation does not change the kernel, since after the integration over the new vertices one reproduces the initial expression. The next transformation is the following. Let us consider the subdiagram formed by the four lines (which have indices $2 i u, \alpha_{u}, \gamma_{u}, 2 s$ ) attached to the right (black) vertex in the middle line of the transformed diagram, and the line with index $\beta_{u}$ which connects the lines with indices $\alpha_{u}$ and $\gamma_{u}$. It can be checked that the indices satisfy the conditions $\alpha_{u}+2 i u=2 s^{\prime}=\gamma_{u}+2 s$ and $\beta_{u}=2 s^{\prime}-\alpha_{u}-\gamma_{u}$. It allows one to use the permutation relation shown in Figure 2 After the transformation, the line with the index $\beta_{u}$ changes its position and will connect the endpoints of the other pair of lines. In addition, the indices of the lines in the new diagrams have to be changed, namely one should interchange $\alpha_{u}$ and $2 s\left(\alpha_{u} \leftrightarrow 2 s\right)$ and $\gamma_{u}$ and $2 i u\left(\gamma_{u} \leftrightarrow 2 i u\right)$.

Next one notices that the subdiagram formed by the lines attached to the next vertex has exactly the same form as the one considered just now. Therefore one can repeat this transformation successively. As the result all lines with index $\beta_{u}$ in the upper part of the diagram change their positions, and one has also to interchange the indices in the way described above, namely, $\alpha_{u} \leftrightarrow 2 s$. Further, since the interchange $2 i u \leftrightarrow \gamma_{u}$ occurs twice for the all lines except for the first and the last one in this chain, the whole effect will be that the line attached to the leftmost vertex (white blob) in the figure will get the index $2 i u$, while all other "horizontal" lines will have the same index, $\gamma_{u}$.

It is important that after this series of transformations only three lines will be attached to the leftmost vertex (white blob in the Figure 9). The integration measure in this vertex corresponds to the conformal spin $s^{\prime \prime}=i u$. Since the incoming arrow has index 2iu, and two other arrows come out of the vertex, one can integrate over this vertex. After the integration the line with index $2 i u$ disappears (see Eq. (3.24) ) and one can easily check that the resulting diagram has the form of the integral kernel for the operator $\mathcal{Q}\left(u+i s_{0}\right) \widetilde{\mathcal{Q}}\left(u-i s_{0}\right)$.

\section{Baxter equation}

In this section we study the relation between $Q$-operators and the transfer matrices over finite dimensional auxiliary spaces. For a negative (half)integer values of the spin of auxiliary space, $s_{0}=-n$, the representation space $\mathbf{V}_{s_{0}=-n}$ contains an invariant subspace $V_{n}$. Thus, the subspace 


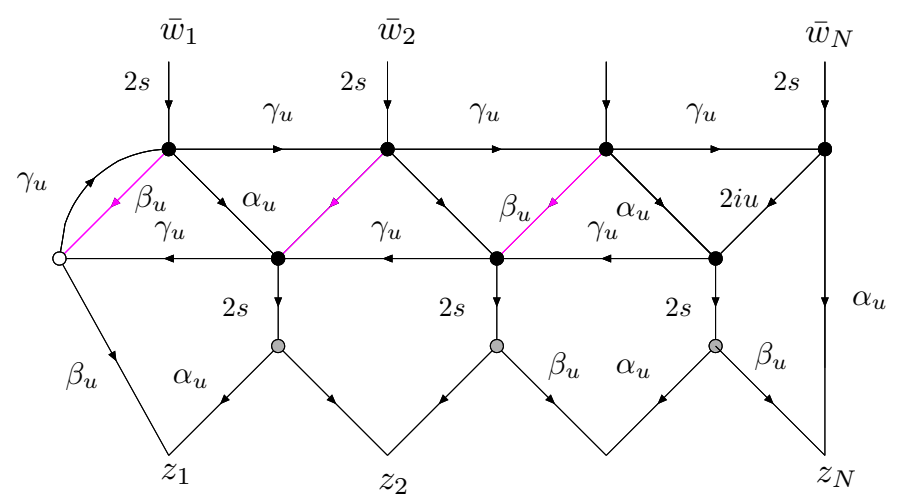

Figure 9. The diagram for the kernel of the transfer matrix for the open spin chain. All notations the same as in Figure 8 The gray dots denote the integration vertices corresponding to the spin $s$.

$\mathbf{V}_{s} \otimes V_{n}$ is an invariant subspace of the $\mathcal{R}_{s s_{0}}$-operator. It has, therefore, the triangular form

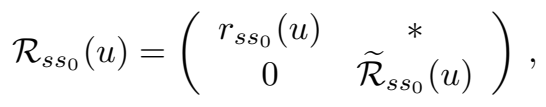

where $r_{s s_{0}}(u)$ is the restriction of the operator $\mathcal{R}_{s s_{0}}$ to the subspace $\mathbf{V}_{s} \otimes V_{n}$. The operator $\widetilde{\mathcal{R}}_{s s_{0}}(u)$ acts on the space $\mathbf{V}_{s} \otimes \mathbf{V}_{s_{0}} / V_{n} \sim \mathbf{V}_{s} \otimes \mathbf{V}_{1+n / 2}$ and satisfies the YB relation (2.1). Therefore it has to be proportional to $\mathcal{R}_{s, s_{0}^{\prime}}(u)$ with the spin $s_{0}^{\prime}=1-s_{0}=1+n$

$$
\widetilde{\mathcal{R}}_{s, s_{0}=-n}(u)=f_{n}(u) \mathcal{R}_{s, s_{0}=1+n}(u) .
$$

The normalization coefficient

$$
f_{n}(u)=(-1)^{2 n+1} \frac{\Gamma(s+1+n-i u)}{\Gamma(s-n-i u)} \frac{\Gamma(s-n+i u)}{\Gamma(s+1+n+i u)}
$$

can be found by comparing the eigenvalues of $\widetilde{\mathcal{R}}_{s s_{0}}(u)$ and $\mathcal{R}_{s, s_{0}^{\prime}}(u)$ on the eigenstates, $\psi_{k}\left(z_{1}, z_{2}\right)=$ $\left(z_{1}-z_{2}\right)^{k}$, or by using the intertwining relation for the $\mathcal{R}^{ \pm}$-operators. The latter takes the form

$$
\begin{aligned}
& \frac{1}{z_{01}^{2 n+1}} \cdot \frac{\Gamma\left(z_{01} \partial_{0}+2\left(s_{0}-\alpha\right)\right)}{\Gamma\left(z_{01} \partial_{0}+2 s_{0}\right)}=\frac{\Gamma\left(z_{01} \partial_{0}+2\left(s_{0}^{\prime}-\alpha^{\prime}\right)\right)}{\Gamma\left(z_{01} \partial_{0}+2 s_{0}^{\prime}\right)} \partial_{0}^{2 n+1}, \\
& \partial_{1}^{2 n+1} \frac{\Gamma\left(z_{10} \partial_{1}+2 s_{0}\right)}{\Gamma\left(z_{10} \partial_{1}+2\left(s_{0}-\alpha\right)\right)}=\frac{\Gamma\left(z_{10} \partial_{1}+2 s_{0}^{\prime}\right)}{\Gamma\left(z_{10} \partial_{1}+2\left(s_{0}^{\prime}-\alpha^{\prime}\right)\right)} \frac{1}{z_{10}^{2 n+1}},
\end{aligned}
$$

where $s_{0}=-n, s_{0}^{\prime}=1+n, \alpha^{\prime}=\alpha+n+1 / 2, n$ being half-integer.

Equations (4.1), (4.2) imply the following relation for the transfer matrices of the closed spin chain

$$
\mathbf{T}_{s_{0}=-n}(u)=t_{n}(u)+\left(f_{n}(u)\right)^{N} \mathbf{T}_{s_{0}=1+n}(u),
$$

where $t_{n}(u)$ is the transfer matrix with finite dimensional auxiliary space, $t_{n}(u)=\operatorname{tr}_{V_{n}} r_{10}(u) \ldots r_{N 0}(u)$. Using the factorization property (3.10) and introducing the notation

$$
\Delta(u) \widetilde{Q}(u)=\bar{Q}(u+i),
$$

where

$$
\Delta(u)=\left(\frac{\Gamma(1-s-i u)}{\Gamma(1+s-i u)}\right)^{N}
$$


we rewrite Eq. (4.6) in the form

$\Delta(u+i n) t_{n}(u)=Q(u-i n) \bar{Q}(u+i(1+n))-Q(u+i(1+n)) \bar{Q}(u-i n)$.

For $n=0$ the transfer matrix $t_{n=0}(u)=1$ and Eq. (4.9) reads

$$
\left(\frac{\Gamma(1-s-i u)}{\Gamma(1+s-i u)}\right)^{N}=Q(u) \bar{Q}(u+i)-Q(u+i) \bar{Q}(u),
$$

which is the Wronskian relation between the operators $Q$ and $\bar{Q}$. Further, one can exclude the operator $\bar{Q}$ (or $Q$ ) from eq. (4.9). Indeed, multiplying both sides by $Q(u-i m)$, where $m$ is (half)integer such that $m+n$ is integer and $-n \leq m \leq n-1$, after some algebra one finds

$$
\begin{aligned}
\tilde{t}_{n}(u) Q(u-i m)= & \tilde{t}_{(n+m) / 2}\left(u+\frac{i(n-m)}{2}\right) Q(u-i n)+ \\
& \tilde{t}_{(n-m-1) / 2}\left(u-\frac{i(n+m+1)}{2}\right) Q(u+i(n+1)),
\end{aligned}
$$

where $\tilde{t}_{n}(u)=\Delta(u+i n) t_{n}(u)$. The same equation holds for the $\bar{Q}$ operator as well. The first relation $(n=-m=1 / 2)$ among the ones in (4.11) is nothing else but the Baxter equation

$$
\tau_{N}(u) Q(u)=(u+i s)^{N} Q(u+i)+(u-i s)^{N} Q(u-i),
$$

where

$$
\tau_{N}(u)=\operatorname{tr} L_{1}(u) \ldots L_{N}(u)
$$

and $L(u)$ is the Lax operator

$$
L(u)=u+i\left(\begin{array}{cc}
S_{0} & S_{-} \\
S_{+} & -S_{0}
\end{array}\right) .
$$

To derive Baxter equation (4.12) from (4.11) one put $n=-m=1 / 2$ and takes into account that $t_{0}(u)=1$ and $t_{1 / 2}(u-i / 2)=(u-i s)^{-N} \tau_{N}(u)$. The latter relation follows from the relation $L(u)=(u-i s) r_{s,-1 / 2}(u-i / 2)$ which can easily be verified by comparison of the eigenvalues 23. It is evident that the operator $\bar{Q}(u)$ satisfies the same equation (4.12). Thus the operators $Q(u)$ and $\bar{Q}(u)$ (and, as consequence, their eigenvalues) represent two independent solutions of the Baxter equation (4.12). It can be shown 9] that the eigenvalues of the operator $Q(u)$ are polynomials in $u$. The eigenvalues of the second operator $\bar{Q}(u)$ are meromorphic functions of $u$ with poles of order $N$ ( $N$ is the length of the chain) at the points $u_{k}=-i(1-s+k), k=0,1,2 \ldots, \infty$.

Next, using the triple relation (4.11) one can derive the "fusion" relations for the transfer matrices. Indeed, multiplying the both sides of Eq. (4.11) by the operator $\widetilde{Q}(v)$ and using the factorized expression for the transfer matrix (3.10) one obtaines the relation which involves three $t$ and $T$ transfer matrices. After the substitution $i(v-u) / 2 \rightarrow s_{0}$ and $(u+v) / 2 \rightarrow u$ it takes form

$$
\begin{aligned}
t_{n}\left(u+i s_{0}\right) T_{s_{0}-\frac{m}{2}} & \left(u-\frac{i m}{2}\right)=t_{\frac{n+m}{2}}\left(u+i s_{0}+\frac{i(n-m)}{2}\right) T_{s_{0}-\frac{n}{2}}\left(u-\frac{i n}{2}\right)+ \\
& f_{n m}\left(u+i s_{0}\right) t_{\frac{n-m-1}{2}}\left(u+i s_{0}-\frac{i(n+m+1)}{2}\right) T_{s_{0}+\frac{n+1}{2}}\left(u+\frac{i(n+1)}{2}\right),
\end{aligned}
$$

with

$$
f_{n m}(u)=\frac{\Delta(u-i(m+1))}{\Delta(u+i n)} .
$$


Similarly, starting from Eq. (4.11) involving $\bar{Q}$ operator and multiplying by $Q(v)$ one gets another identity

$$
\begin{array}{r}
t_{n}\left(u-i s_{0}\right) T_{s_{0}+\frac{m+1}{2}}\left(u-\frac{i(m+1)}{2}\right)=t_{\frac{n-m-1}{2}}\left(u-i s_{0}-\frac{i(n+m+1)}{2}\right) T_{s_{0}-\frac{n}{2}}\left(u+\frac{i n}{2}\right)+ \\
f_{-m-1, n}\left(u-i s_{0}\right) t_{\frac{n+m}{2}}\left(u-i s_{0}+\frac{i(n-m)}{2}\right) T_{s_{0}+\frac{n+1}{2}}\left(u-\frac{i(n+1)}{2}\right) .
\end{array}
$$

For $n=-m=1 / 2$ the relations [4.13), 4.15] take the standard form 23] and relate the transfer matrices with adjacment spins of auxiliary space, $T_{s_{0}}$ and $T_{s_{0} \pm 1 / 2}$.

Next, starting from the Eqs. (4.9) and (4.11) one can derive two quadratic relations for the finite-dimensional transfer matrices $t_{n}(u)$. The first one is

$$
\begin{aligned}
\tilde{t}_{\frac{m-n-1}{2}}\left(u+\frac{i k}{2}\right) \tilde{t}_{\frac{m+n-1}{2}}\left(u-\frac{i k}{2}\right)= & \tilde{t}_{\frac{m-k-1}{2}}\left(u+\frac{i n}{2}\right) \tilde{t}_{\frac{m+k-1}{2}}\left(u-\frac{i n}{2}\right) \\
& +\tilde{t}_{\frac{k-n-1}{2}}\left(u+\frac{i m}{2}\right) \tilde{t}_{\frac{k+n-1}{2}}\left(u-\frac{i m}{2}\right) .
\end{aligned}
$$

Here the numbers $m, k, n$ are all integer or half-integer and $m>k>n$. The second relation is obtained from the first one by changing $n \rightarrow-n$.

The treatment of the open spin chain goes along the same lines. The analog of Eq. (4.9) reads

$(2 i u-1) \Delta_{n}^{2}(u) t_{n}^{\mathrm{op}}(u)=\mathcal{Q}(u-i n) \overline{\mathcal{Q}}(u+i(1+n))-\mathcal{Q}(u+i(1+n)) \overline{\mathcal{Q}}(u-i n)$,

where

$$
\widetilde{\mathcal{Q}}(u)=\frac{1}{s-i+i u}\left(\frac{\Gamma(1+s-i u)}{\Gamma(1-s-i u)}\right)^{2 N} \overline{\mathcal{Q}}(u+i)
$$

and

$$
t_{n}^{\mathrm{op}}(u)=\operatorname{tr}_{V_{n}} r_{10}(u) \ldots r_{N 0}(u) r_{N 0}^{-1}(-u) \ldots r_{10}^{-1}(-u) .
$$

Obviously, the operator $\mathcal{Q}(u)(\overline{\mathcal{Q}}(u))$ for the open chain satisfies the same Eq. 4.11] with $\tilde{t}_{n}(u)=$ $(2 i u-1) \Delta_{n}^{2}(u) t_{n}^{\mathrm{op}}(u)$. The Wronskian relation and Baxter equation take the well known form [22, 13,

$(2 i u-1)\left(\frac{\Gamma(1-s-i u)}{\Gamma(1+s-i u)}\right)^{2 N}=\mathcal{Q}(u) \overline{\mathcal{Q}}(u+i)-\mathcal{Q}(u+i) \overline{\mathcal{Q}}(u)$,

$\tau_{N}^{\mathrm{op}}(u) \mathcal{Q}(u)=\frac{2 i u+1}{2 i u}(u+i s)^{2 N} \mathcal{Q}(u+i)+\frac{2 i u-1}{2 i u}(u-i s)^{2 N} \mathcal{Q}(u-i)$.

Here $\tau_{N}^{\mathrm{op}}(u)=\operatorname{tr} L_{1}(u) \ldots L_{N}(u) L_{N}(u) \ldots L_{1}(u)$. Again, the eigenvalues of the operator $Q(u)$ are polynomials in $u$ [13], while the eigenvalues of $\bar{Q}$ are meromorphic functions.

In full analogy with the closed spin chain one can derive two sets of the "fusion" relations and check that the finite-dimensional transfer matrices, $\tilde{t}_{n}(u)=(2 i u-1) \Delta_{n}^{2}(u) t_{n}^{\mathrm{op}}(u)$, satisfy the quadratic relation 4.16).

\section{Conclusions}

In this paper we considered the quantum spin chains with $s \ell(2)$ symmetry. The Hilbert space of the model is given by the tensor product of the $s \ell(2)$ modules. For a generic spin $s$, the latter are infinite 
dimensional and equivalent to the space of the polynomials of an arbitrary degree, $\mathbf{V}_{s}=\mathbb{C}[z]$. Using the factorization of the $\mathcal{R}$ operator

$$
\mathcal{R}_{12}(u)=P_{12} \mathcal{R}_{12}^{+}\left(\frac{s_{2}-s_{1}+i u}{2}\right) \mathcal{R}_{12}^{-}\left(\frac{s_{1}-s_{2}+i u}{2}\right)
$$

we have shown that the transfer matrices both for the closed and open homogeneous spin chains (for generic spin of the auxiliary space $s_{0}$ ) factorize into a product of two commuting operators $Q$ and $\widetilde{Q}$. The latter are given by the trace of the product of operators $\mathcal{L}_{k 0}^{ \pm}=\mathcal{R}_{k 0}\left(\mp i\left(s_{0}-s\right)\right)$ over the auxiliary space (see Eqs. (3.11), (3.20).

For negative half-integer spins, $s_{0}=-n, n=0,1 / 2,1, \ldots$, the module $\mathbf{V}_{s}$ has a finite dimensional invariant subspace, $V_{n}$. The representation induced on the factor space $\mathbf{V}_{-n} / V_{n}$ is equivalent to the $s \ell(2)$ module with spin $s_{0}^{\prime}=1+n$. We have shown, that the operators $Q$ and $\widetilde{Q}$ satisfy the finitedifference (Baxter) equation, which follows unambiguously from the structure of the reducible $s \ell(2)$ modules and factorization property of the transfer matrices. The treatment of the closed and open spin chains goes along the same lines with minor differences. We hope that similar analysis will be applicable to the spin chains with higher rank symmetry groups.

The factorization property of the transfer matrices breaks down for the finite dimensional spin chain, therefore they require a special consideration. The problem of construction of the Baxter $Q$ operator for the finite dimensional spin chains (in particular $X X X_{1 / 2}$ spin magnet) was considered in the Refs. [10, 24].

\section{Acknowledgments}

The authors are grateful to G. P. Korchemsky for helpful discussions. This work was supported by the RFFI grants 03-01-00837 (S. D. and A. M.) and 05-01-0092 (S. D.) and by the Helmholtz Association, contract number VH-NG-004 (A. M.)

\section{References}

[1] A. V. Belitsky, V. M. Braun, A. S. Gorsky and G. P. Korchemsky, Int. J. Mod. Phys. A 19 (2004) 4715 arXiv:hep-th/0407232.

[2] A. A. Tseytlin, "Spinning strings and AdS/CFT duality," arXiv:hep-th/0311139

[3] L. D. Faddeev, E. K. Sklyanin and L. A. Takhtajan, Theor. Math. Phys. 40 (1980) 688 [Teor. Mat. Fiz. 40 (1979) 194].

[4] L. D. Faddeev, Les-Houches Lectures, 1995, "How Algebraic Bethe Ansatz works for integrable model," arXiv:hep-th/9605187

[5] R. J. Baxter, Exactly Solved Models in Statistical Mechanics, Academic Press, London, 1982.

[6] M. Gaudin and V. Pasquier, J. Phys. A 25 (1992) 5243.

[7] A. Y. Volkov, Lett. Math. Phys. 39 (1997) 313

[8] V. V. Bazhanov, S. L. Lukyanov and A. B. Zamolodchikov, Commun. Math. Phys. 190 (1997) 247

[9] S Derkachov J. Phys. A 32 (1999) 5299

[10] G. P. Pronko, Commun. Math. Phys. 212 (2000) 687

[11] V. B. Kuznetsov, M. Salerno and E. K. Sklyanin, J. Phys. A 33 (2000) 171

[12] M. Rossi, R. Weston J. Phys. A 35 (2002) 10015

[13] S. E. Derkachov, G. P. Korchemsky and A. N. Manashov, JHEP 0310 (2003) 053

[14] M. Kirch and A. N. Manashov, JHEP 0406 (2004) 035

[15] C. Korff, "Representation Theory and Baxter's TQ equation for the six-vertex model.A pedagogical overview. ", arXiv:cond-mat/0411758

[16] S. E. Derkachov, G. P. Korchemsky and A. N. Manashov, Nucl. Phys. B 617 (2001) 375

[17] S. E. Derkachov, "Factorization of R-matrix and Baxter's Q-operator," arXiv:math.qa/0507252

[18] S. E. Derkachov, "Factorization of the R-matrix.I," arXiv:math.qa/0503396

[19] A. Antonov and B. Feigin, Phys. Lett. B 392 (1997) 115 
[20] P. P. Kulish and E. K. Sklyanin, "Quantum spectral transform. Recent developments," Lect. Notes in Physics, 151, (1982), 61.

[21] E. K. Sklyanin, "Quantum inverse scattering method. Selected topics," in "Quantum Group and Quantum Integrable Systems", (Nankai Lectures in Mathematical Physics) ed. Mo-Lin Ge, Singapore, World Scientific, 1992, pp.63-97; arXiv:hep-th/9211111

[22] E. K. Sklyanin, J. Phys. A 21 (1988) 2375.

[23] P.P. Kulish, N.Yu.Reshetikhin and E.K.Sklyanin, "Yang-Baxter equation and representation theory", Lett.Math.Phys. 5 (1981) 393-403

[24] C. Korff, "A Q-operator for the twisted XXX model", arXiv:math-ph/0511022 